\title{
ANÁLISIS DE LAS CONDICIONES DEL RECURSO HIDRICO EN LA QUEBRADA ESCORIAL, PAMPLONA NORTE DE SANTANDER
}

\section{ANALYSIS OF THE CONDITIONS OF THE WATER RESOURCE IN THE ESCORIAL GORGE, PAMPLONA NORTH OF SANTANDER}

\author{
VILLAMIZAR, Y. ${ }^{1}$; RAMÓN, J. D. ${ }^{2}$; LÓPEZ, L. A. ${ }^{3}$ \\ ${ }^{1}$ Ing. Yeisson Villamizar Mendoza, Ingeniero Ambiental. e-mail: \\ yeisonfvm2014@gmail.com
}

${ }^{2}$ PhD. Jarol Derley Ramón Valencia. Ingeniero de Recursos Naturales y del Ambiente. email: jarolramonvalencia@gmail.com

${ }^{3}$ Ing. Lizeth Amparo López Areniz. Ingeniera Química. Jefe de Planta de Tratamiento EMPOPAMPLONA S.A.E.S.P. e-mail: ing.plantas@empopamplonasa.gov.co

\section{Entidad}

Empresa de Servicios Públicos de Pamplona EMPOPAMPLONA S.A.E.S.P

Cra. 6 № 4-65, Pamplona, Norte de Santander. Colombia

Tel: $(+57) 75684200$ 


\section{Resumen}

La contaminación del recurso hídrico proviene de fuentes naturales y antropogénicas que alteran sus propiedades físicas y químicas, por tal razón se debe conocer la fuente de dicha alteración.

Con la finalidad de determinar las características del recurso hídrico de la quebrada Escorial que atraviesa el casco urbano del municipio de Pamplona, se llevó a cabo la caracterización de los parámetros físico químicos. Debido al costo que representa los análisis físicos químicos se decidió realizar la caracterización por medio de muestreos cada 4 horas, durante 3 días. Se seleccionaron dos puntos de muestreo localizados en la desembocadura y parte alta de la microcuenca, obteniendo 6 muestras. Durante cada muestreo se midieron parámetros In Situ y Ex Situ. Como resultado de la caracterización se obtuvo que la quebrada posee cierto grado de contaminación identificado por la presencia de materia orgánica, determinada por medio de los parámetros DQO y DBO5. Esta contaminación proviene de aguas residuales domésticas y agrícolas, otras de las causas es el arrastre de materia orgánica propia del suelo debido a que se presentan procesos de erosión generándose transporte hacia la parte baja. Este proceso aumenta como consecuencia de la escorrentía producida por las precipitaciones.

Identificado el grado de contaminación se procedió a realizar un censo en la zona rural de las quebradas determinándose la ubicación de las viviendas y el número de habitantes. Debido a que los aportes de aguas residuales domesticas realizadas por las familias de la microcuenca se encuentran dispersas se recomienda implementar un sistema de tratamiento en el sitio de origen de acuerdo a lo recomendado en el titulo J del RAS.

Los Sistemas sépticos constan de un tanque séptico y un filtro anaerobio de flujo ascendente (FAFA), el caudal de diseño de cada sistema se calculó conociendo la población, dotación bruta y el coeficiente de retorno. Con la finalidad de disminuir la cantidad de sistemas se agruparon las viviendas más cercanas en un solo tratamiento.

Palabras clave: Vertimiento, $\mathrm{DQO}, \mathrm{DBO}_{5}$, Séptico. 


\begin{abstract}
The contamination of the water resource comes from natural and anthropogenic sources that alter its physical and chemical properties, for which reason the source of said alteration must be known.
\end{abstract}

In order to determine the characteristics of the water resource of the Escorial gorge that crosses the urban area of the municipality of Pamplona, the characterization of the physical-chemical parameters was carried out. Due to the cost of physical chemical analysis, it was decided to carry out the characterization by means of sampling every 4 hours, for 3 days. Two sampling points located at the mouth and upper part of the micro-basin were selected, obtaining 6 samples. During each sampling, In Situ and Ex Situ parameters were measured. As a result of the characterization, it was obtained that the stream has a certain degree of contamination identified by the presence of organic matter, determined by means of the COD and BOD parameters5. This pollution comes from domestic and agricultural wastewater, other causes are the carry-over of organic matter from the soil due to erosion processes occurring, generating transport to the lower part. This process increases as a consequence of the runoff produced by rainfall.

Once the degree of contamination was identified, a census was carried out in the rural area of the streams, determining the location of the dwellings and the number of inhabitants. Due to the fact that the contributions of domestic wastewater made by the families of the micro-basin are dispersed, it is recommended to implement a treatment system at the site of origin according to what is recommended in title $\mathrm{J}$ of the RAS.

Septic systems consist of a septic tank and an anaerobic upflow filter (FAFA), the design flow of each system was calculated knowing the population, gross endowment and the return coefficient. In order to reduce the number of systems, the closest houses were grouped into a single treatment.

Keywords: Shedding, $\mathrm{DQO}, \mathrm{DBO}_{5}$, Septic.

\title{
1. INTRODUCCIÓN
}


El crecimiento demográfico de la población produce la expansión de centros urbanos generando contaminación, producto de la utilización indiscriminada de los recursos naturales (Gutierrez et al, 2016). Uno de los recursos más contaminados es el agua utilizada para todas las actividades antropogénicas en generación de bienes y servicios (Rojas, 2016).

En el caso del municipio de Pamplona ubicado en la parte alta de la cuenca del rio Pamplonita, el recurso hídrico es utilizado en agricultura y consumo humano, estos usos generan su contaminación con residuos de productos agroquímicos y con las aguas residuales domesticas producidas en el casco urbano y en la zona rural (Valencia, 2013; Rivera et al, 2017).

El casco urbano del municipio de Pamplona es atravesado por varias quebradas que han sido canalizadas con la finalidad de disminuir su contaminación, pero esta finalidad no se cumple, debido a que son utilizadas como canales de conducción de aguas residuales domésticas generando su contaminación, en el presente estudio se identificó las características físico químicas de la quebrada Escorial (Aguinaga, 1996; Domínguez, 2015).

La identificación de contaminación del recurso hídrico se llevó a cabo por medio de la caracterización de la quebrada se tomaron muestras durante tres días, obteniéndose un total de seis muestras, posteriormente se realizaron las pruebas de laboratorio para los parámetros fisicoquímicos, permitiendo determinar las características físicas y químicas, del agua que es vertida al rio Pamplonita (Meneses et al, 2017; Gómez et al, 2017).

\section{METODOLOGIA}

La metodología desarrollada en esta investigación es de tipo mixta, y fue desarrollada como se muestra en la figura 1, permitiendo la determinación de las características físicas y químicas de las quebradas que atraviesan el casco urbano del municipio de Pamplona.

Se llevó a cabo una revisión bibliográfica con el objetivo de conocer la información existente para el área de estudio, para la quebrada se seleccionaron los puntos de muestreo para la medición de caudales y determinación de los parámetros físico químicos; cada punto se sitúa en la desembocadura y otro en la parte alta, con la finalidad de observar la diferencia de calidad entre los puntos de muestreos. El presente diagnóstico de la microcuenca determino los factores antropogénicos y naturales que generan la alteración en las características naturales del recurso hídrico (Rodríguez, 2017). 


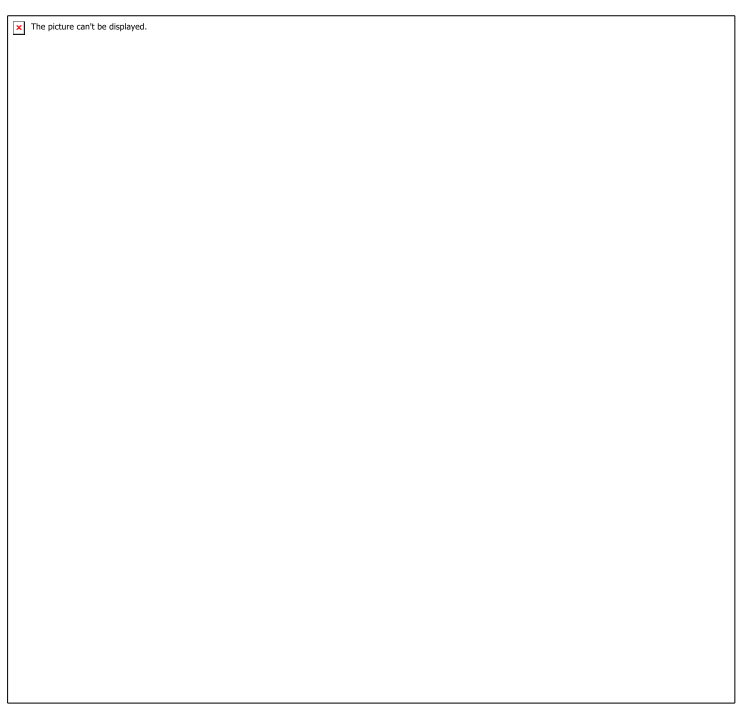

Figura 1. Metodología de investigación.

Fuente: Autor.

Las mediciones de los caudales se realizaron durante tres días a la semana, obteniendo cuatro aforos diarios distribuidos temporalmente en las horas diurnas 6 am, 10 am, 2 pm y 6 pm para la parte alta de la quebrada (punto 2), durante cada aforo se conservó un volumen de muestra para al finalizar el día conformar la muestra por medio de la alícuotas (Gutiérrez \& Romero, 2007). El procedimiento se repitió en el punto 1 con una diferencia de media hora (6:30 am,10:30 am, 2:30 pm y 6:30 pm) lo que posibilito el desplazamiento entre la parte alta de la microcuenca y la desembocadura, logrando obtener dos muestras diarias $y$ un total de seis muestras compuestas para cada quebrada estudiada.

Para la caracterización in situ utilizaron el multiparametro (figura 2) obteniendo los siguientes parámetros In Situ: $\mathrm{Ph}$, Temperatura, Oxígeno disuelto y Conductividad, con el fin de obtener permitiendo tener mayor confiabilidad en los resultados obtenidos. Seguido, se procede a llevar las muestras compuestas al laboratorio de control y calidad, donde se identificó la presencia de sólidos Sedimentables, sólidos totales, sólidos suspendidos totales, sólidos suspendidos volátiles, DQO, DBO, turbiedad, Dureza, Cloruros, Nitritos, Fosfatos, Sulfatos, Color y Alcalinidad. La metodología utilizada para la caracterización Ex Situ se muestran a continuación.

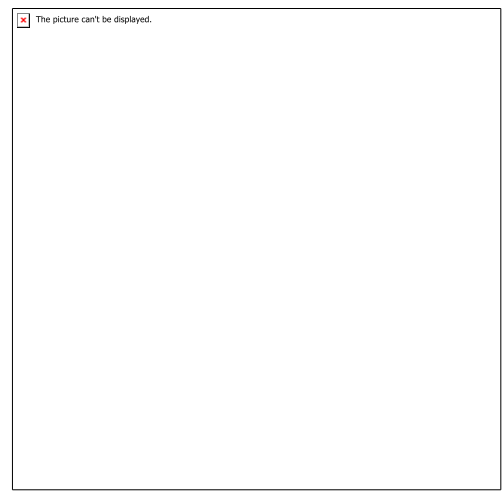

Figura 2. Caracterización In Situ. Fuente: Autor. 
El cálculo de sólidos sedimentables se realizaron pruebas en un cono Imhoff, con un tiempo de sedimentación de 1 hora en condiciones de quietud, el resultado se reporta en $\mathrm{ml} / \mathrm{L}$. Para el cálculo de los sólidos totales la muestra se agito siendo homogenizada, posteriormente se midió entre 10 a $50 \mathrm{ml}$ de muestra $\left(\mathrm{V}_{1}\right)$, siendo transferidos a la capsula de porcelana previamente pesada $\left(\mathrm{W}_{1}\right)$, se deja evaporar la muestra sobre una plancha, se enfría en un desecador y se pesó la capsula $\left(\mathrm{W}_{2}\right)$.

Otro parámetro importante para nuestra investigación son los sólidos suspendidos totales, cuyo procedimiento consiste en pesar el crisol de porcelana con el filtro de fibra de vidrio (W4), posteriormente se filtra de 10 a $20 \mathrm{ml}$ de muestra homogenizada (V2) en el equipo de filtración y se seca en el horno a $105^{\circ} \mathrm{C}$ durante una hora, posteriormente se enfría y pesa (W5). Mediante la ecuación 21 se obtiene el resultado de SST (Figura 3). Además, se calcularon los sólidos suspendidos volátiles, donde se tomaron la capsula de porcelana anterior y se colocó en la mufla previamente calentada a $505^{\circ} \mathrm{C}$ durante una hora, se dejó enfriar y se pesa $\left(\mathrm{W}_{6}\right)$.

Para el cálculo de DQO se agregaron $2 \mathrm{ml}$ de muestra en el tubo de digestión, posteriormente se añadieron $1.5 \mathrm{ml}$ de solución digestora y 3.5 de solución catalizadora (reactivo de ácido sulfúrico). Seguido, se llevó el tubo de digestión al reactor para DQO durante dos horas y se deja enfriar a temperatura ambiente, finalmente se llevó al espectrofotómetro para leer con el programa 480. Otro parámetro que calculamos es la $\mathrm{DBO}_{5}$ aplicando el método manométrico que de acuerdo al resultado obtenido de la DQO se tomaron las cantidades adecuadas en la botella posteriormente se agregaron 3 gotas de inhibidor y 2 lentejuelas de $\mathrm{NaOH}$. Colocando el manómetro en cero se deja incubar durante 5 días, obteniendo al final el consumo de oxígeno, determinando la $\mathrm{DBO}_{5}$ en $\mathrm{mg} / \mathrm{L}$.

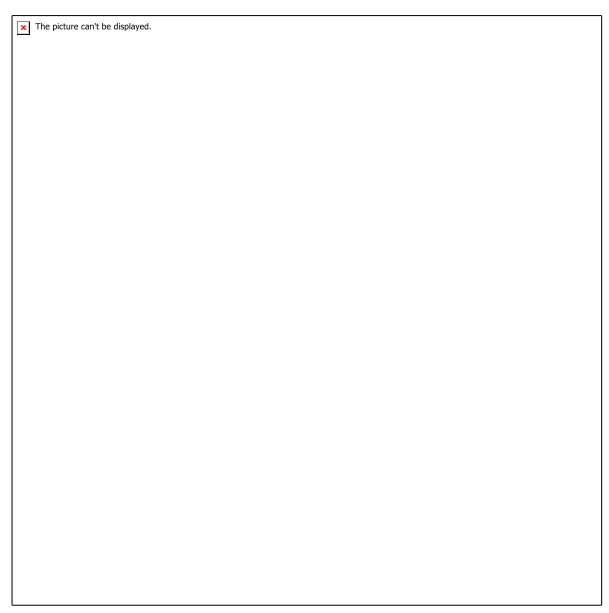

Figura 3. Equipo de filtración.

Fuente: Autor.

Para la determinación de la dureza, se tomaron muestras de $100 \mathrm{ml}$ en un vaso de precipitado y se agregó $0.5 \mathrm{ml}$ de solución buffer y 3 gotas de indicador negro de eriocromo T. Posteriormente se procesaron en el montaje de titulación con 
sustancia titulante EDTA determinando el volumen utilizado.

El Método Argentométrico fue utilizado para determinar cloruros, inicialmente se tomaron una muestra de $100 \mathrm{ml}$ y se agregó $1 \mathrm{ml}$ de solución indicadora, posteriormente se tituló con nitrato de plata hasta observarse un color amarillorosado, determinando el volumen utilizado para calcular los cloruros con la ecuación 24.

Para la determinación de nitritos se tomaron muestras de $10 \mathrm{ml}$ a la que se le agrego 3 gotas de indicador para nitritos y se deja reaccionar durante 20 minutos, posteriormente se llevó al espectrofotómetro donde se lee con el programa 371. Para calcular los fosfatos se tamaron $20 \mathrm{ml}$ de la muestra en un vaso de precipitado, posteriormente se agregó $5 \mathrm{ml}$ de Vanadato Molibdato se homogeniza y se deja reaccionar durante 10 minutos. Se lee en el espectrofotómetro con el programa 490 (Figura 4).

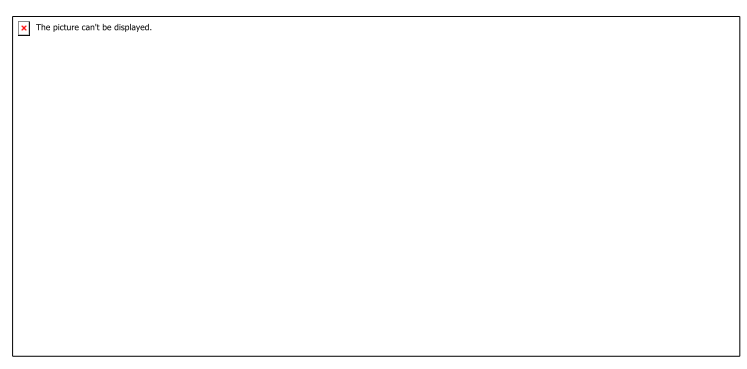

Figura 4. Determinación de nitratos $(A)$ y fosfatos (B). Fuente: Autor.
Para la determinación de Sulfatos, extraen de la muestra de $10 \mathrm{ml}$ se agrega una papeleta de reactivo para sulfatos y se deja reaccionar durante 5 minutos para ser leído en el espectrofotómetro con el programa 680. Otro parámetro identificado es el color donde se tomaron muestras depositadas en la ceda de cuarzo para analizarlas en el fotómetro. Por último, se calcularon la alcalinidad, donde se extrae un volumen de $10 \mathrm{ml}$ de la muestra y se agregaron 3 gotas del indicador de fenolftaleína por medio de la titulación, utilizando como sustancia titulante ácido sulfúrico. Se determinó el volumen de titulante empleado cuando vira a un naranja ladrillo (Morán, 2014).

Otras actividades complementarias de la metodología de investigación son las rutas de trabajo en la microcuenca la Escorial, con la finalidad de identificar actividades antropogénicas, suelos y vegetación, las cuales son causantes de la contaminación identificada en la caracterización. Además, se censaron la población, con la finalidad de conocer que vierten en las inmediaciones de los afluentes determinándose el número de habitantes y viviendas en cada zona (Figura 5). 


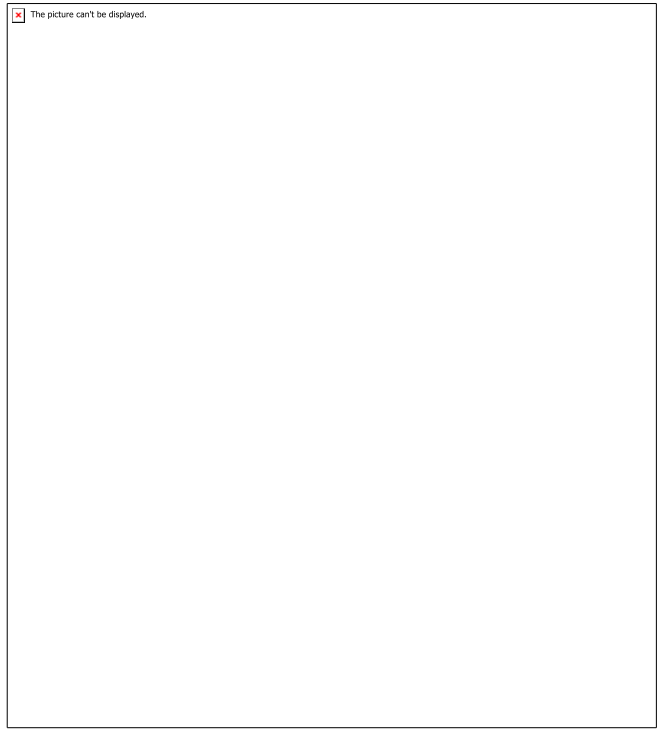

Figura 5. Censo de los habitantes de la zona de estudio. Fuente: Autor.

\section{ANALISIS $Y$ DISCUSIÓN DE RESULTADOS}

La caracterización de la quebrada escorial se realizó en dos puntos de la microcuenca ubicados en la desembocadura (Punto 1) y en la parte media (punto 2) tal como se muestra en la figura 6 , este periodo de muestreo se llevó a cabo durante días con tiempo seco y precipitaciones.

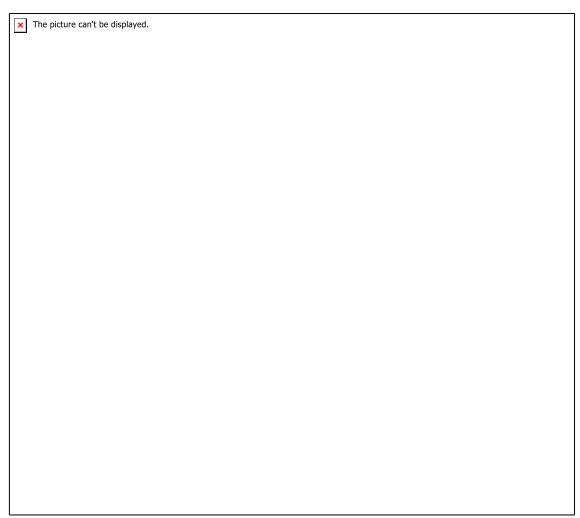

Figura 6. Puntos de Muestreo. Fuente: Autor.

En la tabla 1 se muestran la caracterización IN SITU realizada con el multiparametro identificando que el oxígeno disuelto se encuentran en condiciones estables para los dos puntos de muestreo teniendo valores entre 5-7 $\mathrm{mg} / \mathrm{l}$ en los periodos caracterizados además se identificó que el rango disminuye del punto 2 al 1 debido a que se encontraron aportes de aguas residuales domésticas, por medio de la conductividad se identifica la presencia de sales disueltas con valores inferiores a 280 $\mathrm{mS} / \mathrm{cm}$ lo que nos indica valores favorables en cuanto a calidad de agua, en cuanto al $\mathrm{PH}$ se identificó que se encuentran en los parámetros aceptables.

Tabla 1. Caracterización IN SITU

\begin{tabular}{rrrrr}
\hline Pt & OD & PH & Conductividad & Temperatura \\
\hline 1 & 6.72 & 7.04 & 134.7 & 15.9
\end{tabular}




\begin{tabular}{rrrrr}
\hline 1 & 6 & 7.61 & 137.8 & 18.5 \\
\hline 1 & 6.9 & 7.68 & 125.9 & 17.1 \\
\hline 1 & 7.24 & 7.9 & 103.3 & 16.2 \\
\hline 2 & 7.21 & 7.15 & 83.4 & 15.2 \\
\hline 2 & 7.02 & 7.61 & 84.6 & 16.6 \\
\hline 2 & 7.12 & 7.41 & 81.3 & 16 \\
\hline 2 & 7.24 & 7.03 & 79.3 & 15.9 \\
\hline
\end{tabular}

Fuente: Autor.

El caudal de la microcuenca posee valores promedios entre 3-10 l/s dependiendo de las precipitaciones presentado una variación del $52 \%$ entre el punto de muestreo 1 y 2 debido a los aportes de caudal por escorrentía y tributarios (Franquet, 2005).

En la figura 7 y 8 se observa el comportamiento del caudal para un periodo seco y otro con precipitaciones, identificando su variación para las muestras puntuales tomadas.

Como se observa en la tabla 2 los sólidos totales tuvieron una variación significativa obteniéndose valores entre $50-160 \mathrm{mg} / \mathrm{l}$ para el punto 1 y de $30-70 \mathrm{mg} / \mathrm{l}$ para el punto 2, por el arrastre de materia orgánica a causa de la escorrentía de la Iluvia.

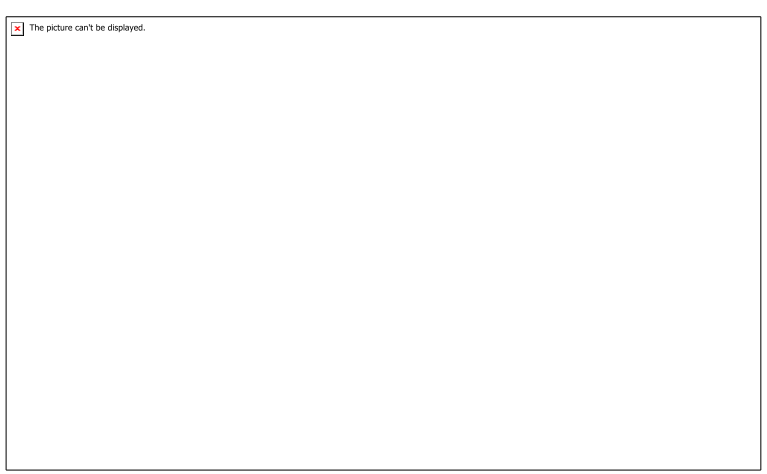

Figura 7. Caudal para periodo seco.

Fuente: Autor.

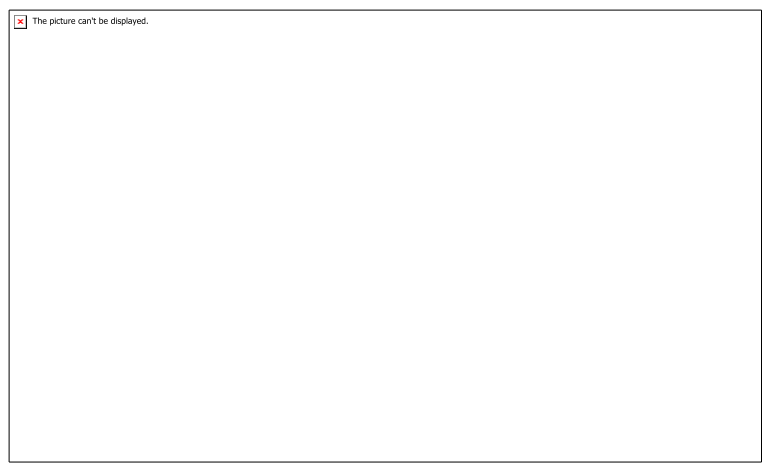

Figura 8. Caudal para periodo seco.

Fuente: Autor.

Tabla 2. Resultados de los sólidos en la quebrada Escorial

\begin{tabular}{cccc}
\hline Pt & $\begin{array}{c}\text { Solidos } \\
\text { Totales } \\
(\mathrm{mg} / \mathrm{L})\end{array}$ & $\begin{array}{c}\text { Solidos } \\
\text { Suspendidos } \\
\text { Totales } \\
(\mathrm{mg} / \mathrm{L})\end{array}$ & $\begin{array}{c}\text { Solidos } \\
\text { Suspendidos } \\
\text { Volátiles } \\
(\mathrm{mg} / \mathrm{L})\end{array}$ \\
\hline 1 & 50 & 30 & 10 \\
\hline 2 & 30 & 20 & 10 \\
\hline 1 & 160 & 130 & 20 \\
\hline
\end{tabular}




\begin{tabular}{llll}
\hline 2 & 70 & 50 & 10 \\
\hline 1 & 110 & 70 & 20 \\
\hline 2 & 70 & 40 & 10
\end{tabular}

Fuente: Autor.

Los sólidos suspendidos totales y volátiles indican que la quebrada Escorial presenta buena calidad del recurso hídrico en los periodos secos caso contrario a lo ocurrido durante los muestreos realizados durante los días con lluvia (Camargo, 2016).

Los resultados obtenidos para la caracterización físico química se muestran en la tabla 3, identificando como resultado que la DQO presenta sus valores mínimos en los días con menor precipitación calificándose como una fuente aceptable con indicios de contaminación para el día 18 de abril, esta clasificación cambia para los días siguientes en los cuales se clasifica como contaminada y fuertemente contaminada a causa del arrastre de material (Blanco, 2010). La turbiedad determinada coincide con los valores de lo solidos registrados en las tablas anteriores (Villamizar \& Justinico, 2017).

Tabla 3. Caracterización Físico Química. Quebrada Escorial

\begin{tabular}{|c|c|c|c|c|c|c|c|c|c|c|}
\hline 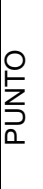 & $\begin{array}{l}\bar{\partial} \\
\text { ఏ } \\
\xi \\
0 \\
0 \\
0 \\
0\end{array}$ & 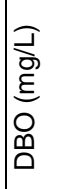 & 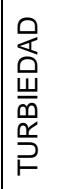 & 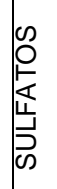 & 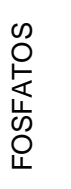 & 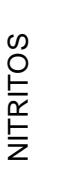 & 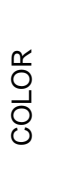 & 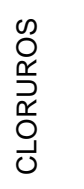 & 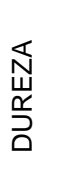 & 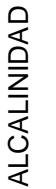 \\
\hline 1 & 27 & 15 & 9.8 & 4 & 0.04 & 0.03 & 103 & 13.99 & 35.5 & 35.0 \\
\hline 2 & 24 & 14 & 6.1 & 3 & 0.02 & 0.01 & 85 & 10.50 & 36.2 & 46.0 \\
\hline 1 & 96 & 81 & 10.3 & 3 & 0.05 & 0.04 & 615 & 14.87 & 36.6 & 44.4 \\
\hline 2 & 58 & 34 & 6.5 & 2 & 0.03 & 0.01 & 91 & 13.99 & 35.3 & 42.0 \\
\hline 1 & 213 & 130 & 20.8 & 4 & 0.04 & 0.08 & 390 & 13.99 & 31.0 & 54.3 \\
\hline 2 & 128 & 80 & 15.3 & 2 & 0.02 & 0.06 & 80 & 13.99 & 43.0 & 52.2 \\
\hline
\end{tabular}

Fuente: Autor.

El recurso hídrico de la quebrada Escorial se clasifica como Dura y muy dura tanto en el punto 1 como en el 2, principalmente por la presencia de magnesio identificado por el color rojizo del agua.

En el censo realizado en la quebrada Escoria se encontró un total de 61 habitantes distribuidos en 14 viviendas tal como se muestra en la tabla 40 , estas familias se dedican a la ganadería y agricultura (Figura 9). 


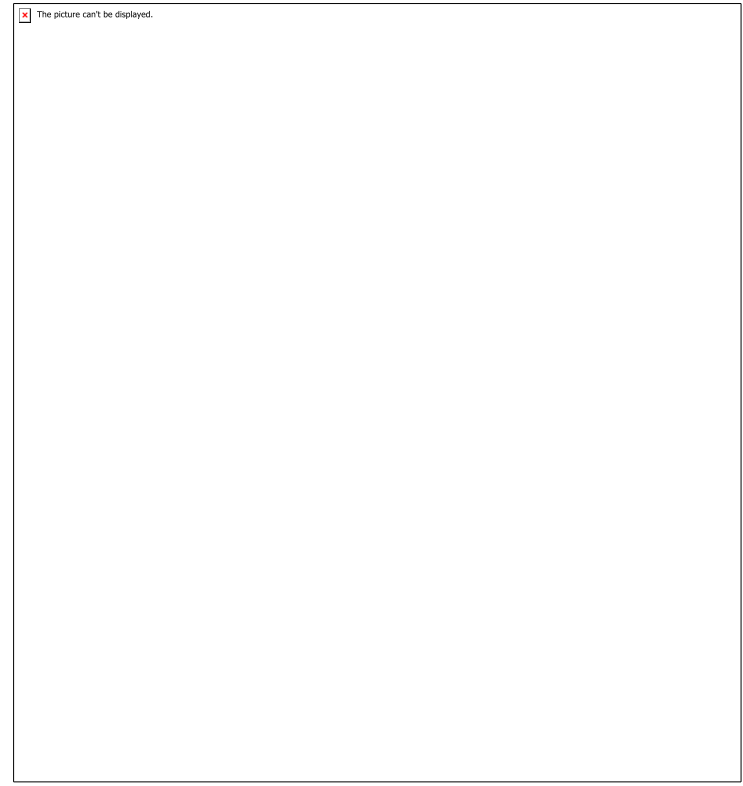

Figura 9. Puntos de contaminación Quebrada Escorial. Fuente: Autor.

Las aguas residuales domesticas son fuente de contaminación del recurso hídrico como solución para mitigar el impacto generado por estas sobre la quebrada escorial se plantea dimensionar sistemas de tratamiento (Hernández \& Sánchez, 2014). Debido a que las viviendas se encuentran en la parte rural, por lo tanto, el número de sistemas de tratamiento de aguas residuales domesticas dependen de la cercanía de las viviendas (Figura 10).

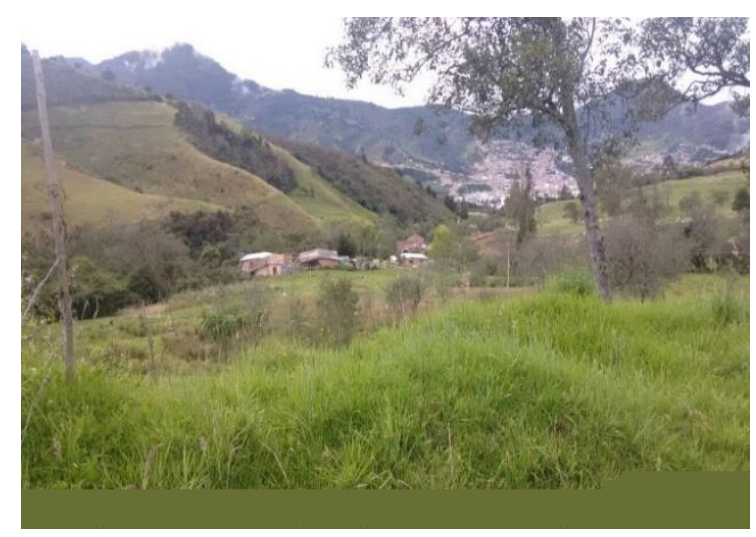

Figura 10. Vivienda alejada del sistema de tratamiento de aguas residuales domésticas.

Fuente: Autor.

Como solución a estas fuentes antropogénicas de contaminación se recomienda implementar un sistema compuesto por un tanque séptico (Tabla 4) y un FAFA (Tabla 5), los cuales cumplirán con el tratamiento de las aguas residuales de la parte rural de la quebrada Escorial (Figura 11) (Fernández, 2010; Aldana, 2017).

Tabla 4. Calculo del Tanque Séptico para el tratamiento aguas residuales.

Parámetro de Diseño Ecuación Resultado

$\begin{array}{lll}\text { Borde Libre } & B l & 0.2 \mathrm{~m} \\ \text { Volumen } & V=Q * T R H & 0.306 \mathrm{~m}^{3} \\ \text { Ancho } & 0.4 \mathrm{~m} \\ \text { Largo } & B=\sqrt{\frac{V}{H_{u} * \frac{l}{b}}} & \\ & L=B * \frac{l}{b} & 0.8 \mathrm{~m} \\ \text { Altura útil corregida } & H_{u}^{*}=\frac{V}{B * L} & 0.96 \mathrm{~m}\end{array}$




\begin{tabular}{|c|c|c|}
\hline $\begin{array}{l}\text { Volumen de } \\
\text { almacenamiento de natas y } \\
\text { espumas }\end{array}$ & $V_{n}=\frac{T_{n} * P * N}{1000}$ & $0.021 \mathrm{~m}^{3}$ \\
\hline Altura de natas & $h_{n}=\frac{V_{n}}{A}$ & $0.066 \mathrm{~m}$ \\
\hline $\begin{array}{l}\text { Altura de los orificio del } \\
\text { primer compartimiento }\end{array}$ & $h_{o}=0.4 * H_{u}^{*}$ & $0.38 \mathrm{~m}$ \\
\hline Largo del compartimiento 1 & $L_{1}=\frac{2}{3} L$ & $0.53 \mathrm{~m}$ \\
\hline Largo del compartimiento 2 & $L_{2}=\frac{1}{3} L$ & $0.27 \mathrm{~m}$ \\
\hline Volumen de lodos & $V_{\text {lod }}=h_{o} * B * L$ & $0.12 \mathrm{~m}^{3}$ \\
\hline Lodos generados & $L o d=P * T l$ & $0.15 \mathrm{~m}^{3} / \mathrm{año}$ \\
\hline Limpieza de lodos & $L_{l o d}=\frac{V_{l o d}}{L o d}$ & 0.82 años \\
\hline Altura Total & $H=H_{u}^{*}+h_{n}+B$ & 1.22 \\
\hline
\end{tabular}

Fuente: Autor.

Tabla 5. Cálculos del FAFA para el tratamiento aguas residuales.

Parámetro de Diseño Ecuación

Resultado

Volumen

$$
V_{F A F A}=0.05 * P \quad 0.15 \mathrm{~m}^{3}
$$

Largo

$$
L_{F A F A}=\frac{V_{F A F A}}{B * H_{F A F A}} 0.75 \mathrm{~m}
$$

TRH

$$
T R H=\frac{Q}{V_{F A F A}} \quad 0.49 \text { días }
$$

Fuente: Autor.

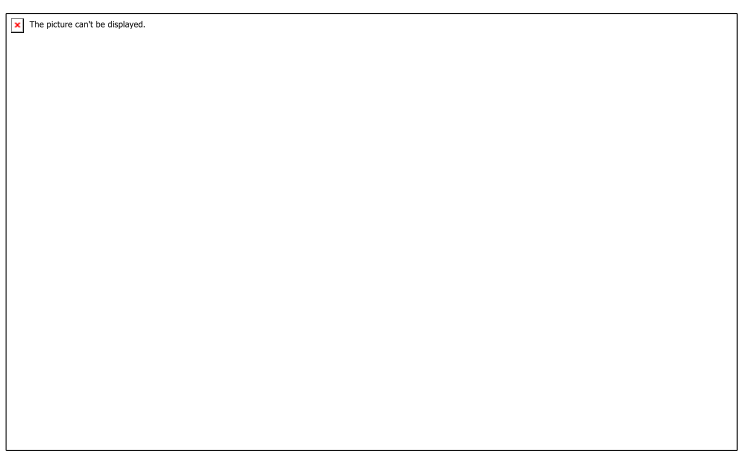

Figura 11. Sistema de Tratamiento 2. Quebrada Escorial. Fuente: Autor.

\section{CONCLUSIONES}

La contaminación de las fuentes hídricas caracterizadas proviene de fuentes antropogénicas como los son las aguas residuales domésticas y actividades agrícolas y ganaderas. Otras son las fuentes naturales como la composición de los suelos y fenómenos de erosión que aportan materia orgánica presente en los horizontes del suelo.

Se observaron que las altas cargas de contaminación registradas en la quebrada escorial son producto del arrastre de material desde la parte alta de la microcuenca producto de la escorrentía generada por las precipitaciones, además, existe un aliviadero del alcantarillado que vierte sobre esta, por lo tanto, parte de la carga orgánica identificada proviene del exceso de aguas residuales vertida en época de lluvias. 
Para el tratamiento de las aguas, se seleccionaron el tanque séptico y el FAFA, debido a que ofrecen eficiencias entre el $70 \%$ y $80 \%$; además se evitara la infiltración de estas aguas residuales, sin previo tratamiento.

\section{Referencias Bibliográficas}

Aguinaga, S. (1996). Manual de procedimientos analíticos para aguas y efluentes. Documento en línea. [Consultado 2017, enero 27]. Disponible en: imasd.fcien.edu.uy/difusion/educa mb/docs/pdfs/manual_dinama.pdf.

Aldana. 2017. Lodos Activados. Parámetros de diseño de lodos activados.

Angulo, W. J., Mendoza, J. A. y Uriel, H. U. (2017). Análisis de la vulnerabilidad por fenómenos de remoción en masa en la Cuenca Tanauca estudio de caso. Revista Ambiental Agua, Aire y Suelo. ISSN 1900-9178. Volumen (8), Numero

DOI: https://doi.org/10.24054/1900 9178.v2.n2.2017.3276

Blanco, A. K. \& León, D. L. (2010). Diseño Hidráulico de La Planta de Tratamiento de Aguas Residuales Para el Municipio de Guacamaya. Bucaramanga. Trabajo de grado (ingenieros Químicos). Universidad Industrial de Santander. Facultad de Ingenierías Fisicoquímicas. Escuela de ingeniería Química.

Camargo, W. C. (2016). Modelación Hidrologico-Hidraulica De Eventos De Inundación En El Rio Bogotá (Sector Tocanzipa-Chia) Usando HEC-RAS. Revista Ambiental Agua, Aire y Suelo. Volumen (7), Numero

DOI: https://doi.org/10.24054/1900

9178.v2.n2.2016.3267

Decreto 2811 de 1974. (1974). Código Nacional de Recursos Naturales Renovables y de Protección al Medio Ambiental. Colombia: Ministerio de Agricultura.

Decreto 1541 de 1978. (1978).Reglamenta la parte III del libro II del decretoley 2811 de 1974: "de las aguas no marítimas" y parcialmente la ley 23 de 1973. Colombia: Ministerio de Agricultura.

Decreto 155 de 2004. (2004). Por la cual se reglamenta el artículo 43 de la ley 99 de 1993 sobre tasa por utilización de aguas y su adoptan otras disposiciones. Colombia: Ministerio de Ambiente, Vivienda y Desarrollo Territorial.

Domínguez, R. (2015). Diseño de una Planta de Tratamiento de Aguas Residuales Urbanas para Poblaciones entre 20 y 25 Mil 
Habitantes. Leganés. Trabajo de Grado (ingeniera en tecnologías industriales). Universidad Carlos III de Madrid. Departamento de ingeniería térmica y fluidos.

Hernández, T. D. \& Sánchez, J. (2014). Diseño de una Planta de Tratamiento de Agua Residual Para el Municipio de San MarcosDepartamento de Sucre. Bogotá. Trabajo de Grado (Ingeniero Civil). Universidad Católica de Colombia. Facultad de ingeniería. Programa de Ingeniería Civil.

Fernández, E. (2010). Proyecto Ejecutivo de Planta de Tratamiento de Aguas Residuales ara la Localidad de Xochiapa, Ver. Xalapa. Tesis (ingeniero Civil). Universidad Veracruzana. Facultad de ingeniería Civil.

Franquet, J. M. (2005). ¿Qué es una cuenca hidrográfica? .Documentó en línea. [Consultado 2017, febrero 23]. Disponible en: http://www.eumed.net/librosgratis/2005/jmfb-h/1u.htm.

Gómez, M. (2017). Características del agua. Documento en línea. 2010. [Consultado 2017. Febrero 25]. Disponible en: http://apoyoambiental.bligoo.com.c o/media/users/19/973332/files/2257 66/CaracteristicasAgua.pdf.
Gómez, S. A., Mora, W. J. y Ramón, B. (2017). Caracterización física y química de la fibra natural de esparto (juncus ramboi subsp. colombianus) como alternativa de refuerzo en materiales compuestos. Revista Ambiental Agua, Aire y Suelo. ISSN 19009178. Volumen (8), Numero (1). DOI: https://doi.org/10.24054/1900 9178.v1.n1.2017.3273

Gutiérrez, H. A. \& Romero, F. (2007). Diseño de la Planta de Tratamiento de Aguas Residuales del Municipio de Macaravita- Departamento de Santander. Bucaramanga. Trabajo de grado (Especialista en ingeniería ambiental). Universidad Industrial de Santander. Facultad de Ingenierías Fisicoquímicas. Escuela de ingeniería Química. Especialización en ingeniería Ambiental.

Gutierrez, T., Castellanos, C. y Hernández, N. (2016). El ordenamiento territorial frente a las consecuencias de los cambios climáticos. Revista Ambiental Agua, Aire y Suelo. ISSN 19009178. Volumen (7), Numero (2). DOI: https://doi.org/10.24054/1900 9178.v2.n2.2016.3338

Ivanova, Y. y Sarmiento, A. (2014). Evaluación de la huella hídrica de la ciudad de Bogotá como una herramienta de la gestión del agua en el territorio urbano. Revista 
Ambiental Agua, Aire y Suelo. ISSN 1900-9178. Volumen (4), Numero (2), 1-5.

DOI: https://doi.org/10.24054/1900 9178.v2.n2.2013.427

Melo, J., Saavedra, S. y Ramón, J. A. (2017). Evaluación de la adsorcion de $C_{+}+2$ y azul de metileno en biosorbentes de bajo costo obtenidos a partir de biomasa residual de la agroindustria de cítricos. Revista Ambiental Agua, Aire y Suelo. ISSN 1900-9178. Volumen (8), Numero (2). DOI: https://doi.org/10.24054/1900 9178.v2.n2.2017.3277

Meneses, V. B., Álzate, D. y Mosquera, J. (2016). Sistema de optimización de las técnicas de planificación en agricultura de precisión por medio de drones. Revista Ambiental Agua, Aire y Suelo. ISSN 19009178. Volumen (7), Numero (2). DOI: https://doi.org/10.24054/1900 9178.v2.n2.2016.3268

Morán, D. (2014). Diseño de Planta de Tratamiento de Aguas Residuales para el Municipio de San Juan Chamelco, Alta Verapaz. Quetzaltenango. Tesis de grado (Ingeniero Ambiental). Universidad Rafael Landívar. Facultad de Ciencias Ambientales y Agrícolas. Licenciatura en ciencias ambientales con énfasis en gestión ambiental.
Moreno, C. y Rueda, L. (2016). La educación ambiental como herramienta para la recuperación de la cobertura vegetal, mediante prácticas agro-ecológicas en la comunidad minera asograstorres, asociación de gravilleros de Sabana de Torres. Revista Ambiental Agua, Aire y Suelo. ISSN 1900-9178. Volumen (7), Numero

DOI: https://doi.org/10.24054/1900 9178.v1.n1.2016.3260

Rojas, J. S. (2016). Aplicación del modelo estocástico WIENER-FPK en caudales medios del rio fonce en condiciones del sistema de Pearson. Revista Ambiental Agua, Aire y Suelo. ISSN 1900-9178. Volumen (7), Numero (1). DOI: https://doi.org/10.24054/1900 9178.v1.n1.2016.3256

Reglamento técnico del Sector de Agua Potable y Saneamiento Básico RAS- 2000. Seccion II. Título E. Tratamiento de Aguas Residuales. Ministerio de Desarrollo Económico Dirección de Agua Potable y Saneamiento Básico.

Resolución 1096 de 2000. (2000). Por la cual se adopta el Reglamento Técnico para el sector de Agua Potable y Saneamiento BásicoRAS. Colombia: Ministerio de Desarrollo Económico. 
Reglamento técnico del Sector de Agua Potable y Saneamiento Básico RAS- 2010.Título J. Alternativas Tecnológicas en agua y Saneamiento para el Sector Rural. Ministerio Ambiente, Vivienda y Desarrollo Territorial.

Resolución 0330 de 2017. (2017). Por la cual se adopta el Reglamento Técnico para el Sector de Agua Potable y Saneamiento Básico (RAS) y se derogan las Resoluciones números 1096 de 2000, 0424 de 2001, 0668 de 2003, 1459 de 2005, 1447 de 2005y 2320 de 2009. Ministerio de Vivienda, Ciudad y Territorio.

Rivera, H. U., Castellanos, C. y Ibarra, A. (2017). Caracterización y cuantificación de los residuos sólidos realizado en el municipio de pamplona, $\mathrm{N}$ de $\mathrm{S}$, Colombia. Revista Ambiental Agua, Aire y Suelo. ISSN 1900-9178. Volumen (8), Numero

DOI: https://doi.org/10.24054/1900

9178.v2.n2.2017.3280

Rodríguez, Y. A. (2017). Conservación de humedales en el marco de gestión de cuencas hidrográficas. Puerto Rondón-Arauca. Revista Ambiental Agua, Aire y Suelo. ISSN 19009178. Volumen (8), Numero (2). DOI: https://doi.org/10.24054/1900 9178.v2.n2.2017.3281

Valencia, A. (2013). Diseño de un Sistema de Tratamiento para las Aguas Residuales de la Cabecera Parroquial de San Luis- Provincia de Chimborazo. RioBambaEcuador. Trabajo de Grado (Ingeniero en Biotecnología Ambiental). Escuela Superior Politécnico de Chimborazo. Programa de Ingeniería en Biotecnología Ambiental.

Villamizar, V. A. y Justinico, A. J. (2017). Reconstrucción paleoclimatica y paleoambiental de los territorios de la llanura inundable del araucana a partir del análisis de sedimentos recientes, Departamento de Arauca. Revista Ambiental Agua, Aire y Suelo. ISSN 1900-9178. Volumen (8), Numero (1). DOI: https://doi.org/10.24054/1900 9178.v1.n1.2017.3272 